\title{
SIMPLE PROOF OF A THEOREM ON PERMANENTS
}

\author{
by D. ž. DJOKOVIĆ $\dagger$
}

(Received 27 December, 1967)

Let $A=\left(a_{i j}\right)$ be an $n \times n$ complex matrix. The permanent of this matrix is

$$
\operatorname{per} A=\sum_{\rho} \prod_{i=1}^{n} a_{i, \rho(i)}
$$

where the sum is taken over all permutations $\rho$ of the set $\{1, \ldots, n\}$.

In a recent paper [1] E. H. Lieb proved an interesting theorem (see below) which he applied to verify some conjectures of M. Marcus and M. Newman. The purpose of this note is to give a simple proof of Lieb's theorem. $\$$

THEOREM. Let $A=\left(a_{i j}\right)$ be an $n \times n$ hermitian positive semidefinite (h.p.s.d.) matrix partitioned as follows

$$
A=\left(\begin{array}{ll}
B & C \\
C^{*} & D
\end{array}\right)
$$

where $B$ is a $p \times p$ matrix. In addition suppose that $A$ does not have a zero row. Let $A(\lambda)$ be the $n \times n$ matrix obtained from $A$ by replacing $B$ by the matrix $\lambda B, \lambda$ being a complex number. Then all the coefficients of the pth degree polynomial $P(\lambda)=\operatorname{per} A(\lambda)$ are real and non-negative. Furthermore, if $B$ and $D$ are positive definite (p.d.) then the coefficient $c_{t}$ of $\lambda^{t}$ in $P(\lambda)$ is zero if and only if all subpermanents of $C$ of order $p-t$ vanish.

Proof. Let

$$
\begin{array}{lll}
\alpha=\left(\alpha_{1}, \ldots, \alpha_{p}\right), & \alpha^{\prime}=\left(\alpha_{1}, \ldots, \alpha_{t}\right), & \alpha^{\prime \prime}=\left(\alpha_{t+1}, \ldots, \alpha_{p}\right), \\
\sigma=\left(\sigma_{1}, \ldots, \sigma_{n-p}\right), & \sigma^{\prime}=\left(\sigma_{1}, \ldots, \sigma_{p-t}\right), & \sigma^{\prime \prime}=\left(\sigma_{p-t+1}, \ldots, \sigma_{n-p}\right),
\end{array}
$$

where $\alpha$ and $\sigma$ are permutations of the sets $\{1, \ldots, p\}$ and $\{p+1, \ldots, n\}$, respectively. When we need more such sequences we shall use the letters $\beta$ and $\tau$ instead of $\alpha$ and $\sigma$, respectively.

The coefficient $c_{t}$ is the sum of all permutation products

$$
\prod_{i=1}^{n} a_{i, \rho(i)}
$$

which contain $t$ elements of $B, p-t$ elements of $C, p-t$ elements of $C^{*}$ and $n-2 p+t$ elements of $D$. We clearly have

$$
M c_{t}=\sum_{\alpha, \beta, \sigma, \tau} a_{\alpha^{\prime} \beta^{\prime}} a_{\alpha^{\prime \prime} \tau^{\prime}} a_{\sigma^{\prime} \beta^{\prime \prime}} a_{\sigma^{\prime \prime} \tau^{\prime \prime}}
$$

† This work was supported in part by the NRC Grant No. A-5285.

W We mention that the theorem in [1] contains an inaccuracy concerning the conditions under which $c_{t}=0$. We present a corrected formulation. The proof given in [1] concerning the conditions under which $c_{p-1}>0$ is still valid and so is the addendum on p. 129. 
where $M=t !(p-t) !^{2}(n-2 p+t) !$ and, for instance,

$$
a_{\alpha^{\prime} \beta^{\prime}}=\prod_{i=1}^{t} a_{\alpha_{t}, \beta_{i}} \cdot
$$

Since $A$ is h.p.s.d. there exist $n$ vectors $f^{i}(i=1, \ldots, n)$ such that

$$
a_{i j}=\left(f^{i}, f^{j}\right)=\sum_{k=1}^{n} \overline{f_{k}^{i}} f_{k}^{j}
$$

where $f_{k}^{i}(k=1, \ldots, n)$ are coordinates of $f^{i}$. The bar denotes the complex conjugate. Using this representation of $a_{i j}$ we get

$$
M c_{t}=\sum_{\alpha, \beta, \sigma, \tau} \sum_{I, J, K, R} \bar{F}_{I}^{\alpha^{*}} F_{I}^{\beta^{\prime}} \bar{F}_{J}^{\alpha^{\prime \prime}} F_{J}^{\tau^{\prime}} \bar{F}_{K}^{\sigma^{\prime}} F_{R}^{\beta^{\prime \prime}} \bar{F}_{R}^{\sigma^{\prime \prime}} F_{R}^{\tau^{\prime \prime}}
$$

where, for instance,

$$
F_{I}^{\alpha^{\prime}}=\prod_{s=1}^{t} f_{i_{s}}^{\alpha_{*}}
$$

The letters $I, J, K, R$ denote the sequences of indices $I=\left(i_{1}, \ldots, i_{t}\right), J=\left(j_{1}, \ldots, j_{p-t}\right)$, $K=\left(k_{1}, \ldots, k_{p-i}\right), R=\left(r_{1}, \ldots, r_{n-2 p+i}\right)$. The sum over $I$, for instance, means the sum over all indices $i_{1}, \ldots, i_{t}$. Each index runs through the values $1, \ldots, n$.

Changing the order of summation, we get

$$
\begin{aligned}
M c_{t} & =\sum_{I, R}\left[\sum_{J}\left(\sum_{\alpha} \bar{F}_{I}^{\alpha^{\prime}} \bar{F}_{J}^{\alpha^{\prime \prime}}\right)\left(\sum_{\tau} F_{J}^{\tau^{\prime}} F_{R}^{\tau^{\prime \prime}}\right)\right]\left[\sum_{K}\left(\sum_{\beta} F_{I}^{\beta^{\prime}} F_{K}^{\beta^{\prime \prime}}\right)\left(\sum_{\sigma} \bar{F}_{R}^{\sigma^{\prime}} \bar{F}_{R}^{\sigma^{\prime \prime}}\right)\right] \\
& =\sum_{I, R}\left|\sum_{J}\left(\sum_{\alpha} \bar{F}_{I}^{\alpha^{\prime}} \bar{F}_{J}^{\alpha^{\prime \prime}}\right)\left(\sum_{\tau} F_{J}^{\mathfrak{\tau}^{\prime}} F_{R}^{\mathbf{\tau}^{\prime \prime}}\right)\right|^{2} .
\end{aligned}
$$

Hence $c_{s} \geqq 0$. We have $c_{t}=0$ if and only if .

i.e.,

$$
\sum_{J}\left(\sum_{\alpha} \bar{F}_{I}^{\alpha^{\prime}} \bar{F}_{J}^{\alpha^{\prime \prime}}\right)\left(\sum_{\mathbf{\tau}} F_{J}^{\mathfrak{\tau}^{\prime}} F_{R}^{\mathbf{z}^{\prime \prime}}\right)=0
$$

$$
\sum_{\alpha, \tau} \bar{F}_{I}^{\alpha^{\prime}} F_{R}^{\tau^{\prime \prime}} a_{\alpha^{\prime \prime} \tau^{\prime}}=0
$$

for all $I$ and $R$. After summation over $\alpha^{\prime \prime}$ and $\tau^{\prime}$ this condition becomes

$$
\sum_{\alpha^{\prime}, \tau^{\prime \prime}} \bar{F}_{I}^{\alpha^{\prime}} F_{R}^{\tau^{\prime \prime}} \operatorname{per} C\left(\alpha^{\prime}, \tau^{\prime \prime}\right)=0 .
$$

Here $C\left(\alpha^{\prime}, \tau^{\prime \prime}\right)$ denotes the submatrix of $C$ which remains after deleting the rows $\alpha^{\prime}$ and the columns $\tau^{\prime \prime}$ of $A$.

It is obvious that (1) is satisfied if all subpermanents of $C$ of order $p-t$ vanish. Conversely, if $B$ and $D$ are p.d. we shall prove that (1) implies that all these subpermanents vanish. Let $\beta$ and $\sigma$ be arbitrary. Since $B$ is p.d. the vectors $f^{k}(k=1, \ldots, p)$ are linearly independent. Let $g^{k}(k=1, \ldots, p)$ be their reciprocal system of vectors. Similarly, let the system of vectors $g^{k}(k=p+1, \ldots, n)$ be reciprocal to the system $f^{k}(k=p+1, \ldots, n)$. Then we have 


$$
\begin{aligned}
\sum_{I} G_{I}^{\beta^{\prime}} \bar{F}_{I}^{\alpha^{\prime}} & = \begin{cases}1 & \text { if } \beta^{\prime}=\alpha^{\prime}, \\
0 & \text { otherwise, }\end{cases} \\
\sum_{R} \bar{G}_{R}^{\sigma^{\prime \prime}} F_{R}^{\tau^{\prime \prime}} & = \begin{cases}1 & \text { if } \sigma^{\prime \prime}=\tau^{\prime \prime}, \\
0 & \text { otherwise. }\end{cases}
\end{aligned}
$$

Multiplying (1) by $G_{I}^{\beta^{\prime}} \bar{G}_{R}^{\sigma^{\prime \prime}}$ and summing over $I$ and $R$, we get per $C\left(\beta^{\prime}, \sigma^{\prime \prime}\right)=0$. This proves the theorem.

I am grateful to Professor E. H. Lieb for the remark that it is sufficient to suppose that $B$ and $D$ are p.d. ( $A$ may be merely h.p.s.d.).

\section{REFERENCE}

1. E. H. Lieb, Proofs of some conjectures on permanents, J. Mech. Math. 16 (1966), 127-134.

Faculty of Mathematics

UNIVERSITY OF WATERLOO,

Waterloo, Ontario, Canada 